EDITORIAL

\title{
Catheter ablation for atrial fibrillation
}

\author{
P Jais, P Sanders, L F Hsu, M Hocini, M Haissaguerre
}

Heart 2005;91:7-9. doi: 10.1136/hrt.2003.030205

\section{Recent advancements in our understanding of atrial fibrillation have led to the development of catheter ablation techniques that feasibly could achieve a cure for AF}

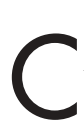
atheter ablation for atrial fibrillation (AF) was first reported 10 years ago in 1994. ${ }^{12}$ Over this relatively short period of time, our strategies and techniques for $\mathrm{AF}$ ablation have dramatically evolved in parallel with improved understanding of the mechanisms initiating and maintaining AF.

Initial approaches to ablation were based on the concept that AF was maintained by multiple wavelet re-entry that required a critical mass of atrial tissue to be sustained. The objective of these procedures was to segment the atria in order to reduce the available atrial tissue that could sustain re-entry, thereby preventing the maintenance of AF. These procedures deployed long linear lesions in the right, left or both atria, replicating the MAZE procedure. ${ }^{23}$ While this approach had limited applicability because of the associated morbidity and outcomes, it revealed the existence of foci, predominantly clustered in pulmonary veins $(\mathrm{PV})$, which were responsible for initiation of $\mathrm{AF}^{4{ }^{5}}$ As a result, most procedures to cure AF now focus on the PV and the prevention of the interaction of these foci with the atrial substance. A variety of approaches have been propounded around this central theme, differing mainly by the localisation tools used to facilitate the procedure. The experience published by pioneering centres over the last 2-3 years is extremely encouraging, with success rates in the absence of antiarrhythmic drugs of $72-90 \%$ and an acceptably low incidence of complications. ${ }^{6-10}$

Nevertheless, as for any treatment, an important determinant of the wider applicability of these techniques is their reproducibility by nonpioneering centres. In this respect, the series presented by Bourke and colleagues ${ }^{11}$ in this issue of Heart, reporting their initial experience in $\mathrm{AF}$ ablation at a non-pioneering centre, is important. The 100 consecutive patients reported have many demographic characteristics that are typically seen in patients referred for AF ablation, a 4:1 male preponderance, and a mean age of 52 years. However, intriguingly this initial experience included a large percentage of patients with persistent AF (64\%), a subset that is perhaps not ideal when commencing $\mathrm{AF}$ ablation as, compared to paroxysmal AF, they are more difficult to ablate. With this in mind centres beginning AF ablation should take into account previous reports and focus (at least initially) on patients with paroxysmal AF. ${ }^{70}$ Commencing with patients in whom less extensive ablation results in the best outcomes facilitates familiarity with the procedure and allows the easier transition during an anticipated period of learning. However, despite their inclusion of a large percentage of patients with persistent $\mathrm{AF}$, Bourke and colleagues ${ }^{11}$ report that $55 \%$ of patients with persistent AF remained in sinus rhythm without the use of antiarrhythmic drugs at six month follow up. In addition, patients with paroxysmal AF achieved a 70\% success rate without antiarrhythmic drugs, remarkably similar to the success rate reported by more experienced centres, thereby demonstrating the reproducibility of this approach.

\section{WHY PULMONARY VEIN ISOLATION IS FREQUENTLY SUCCESSFUL}

$\mathrm{PV}$ isolation was initially advocated on the basis of isolating the dominant source of triggers initiating AF. However, several investigators have suggested that these structures may also have a role in the maintenance of AF. It is recognised that there are some patients with "focal $\mathrm{AF}$ " in whom a rapidly firing focus within the PV was capable of maintaining $\mathrm{AF}^{4}$ In addition, others have observed paroxysmal short cycle length activity within the PV with a distal to proximal activation sequence and have suggested the role of such periodic bursts of activity from the PV in the maintenance of AF. ${ }^{12-15}$

In patients with paroxysmal $\mathrm{AF}$, when $\mathrm{PV}$ isolation is performed during $\mathrm{AF}$, a progressive lengthening of the AF cycle length was observed distant to the ablation site (within the coronary sinus), culminating in the interruption of AF. In addition, following $\mathrm{PV}$ isolation, $\mathrm{AF}$ was no longer inducible in $57 \%$ of patients with paroxysmal AF, despite an aggressive protocol of burst pacing to refractoriness at maximum output from three atrial sites. These observations implicate the PV and the neighbouring atrial tissue in the substrate maintaining $\mathrm{AF}$ in these patients and contribute to the benefits observed with PV ablation, underscoring the importance of PV ablation in any strategy of AF ablation.

\section{WHAT IS THE BEST STRATEGY FOR CATHETER ABLATION OF AF?}

There is a general consensus that $\mathrm{PV}$ ablation is central to any strategy of AF ablation. It is also agreed that the ablation lesions around the PV should be deployed within the atrium, probably at least $1 \mathrm{~cm}$ from the PV ostia. Such a strategy

Abbreviations: $A F$, atrial fibrillation; AFFIRM, atrial fibrillation follow-up investigation of rhythm management; $\mathrm{PV}$, pulmonary vein 
has the benefit of including atrial foci at the PV-left atrial junction in the lesion and is very likely to reduce the risk of PV stenosis. A variety of localisation systems (CARTO, LocaLisa, NavX, RPM, EnSite, ICE) can facilitate the deployment of atrial lesions and reduce the procedure duration. ${ }^{6716}$ However, none of these systems can offer an absolute guarantee of avoiding ablation within the PV to reduce the risk of stenosis.

More debatable is the need for complete electrical isolation of the PV. The anatomical approach advocated by Pappone and colleagues has provided excellent results in specialised centres but has not been reproducible by all groups. ${ }^{717}$ Such anatomical ablation is associated with only a $40-50 \%$ electrical isolation of the PVs. We strongly believe that complete isolation cannot be worse than incomplete isolation, and that isolation should provide a better long term outcome for our patients-a concept supported historically by the ablation of many other arrhythmias. For example, before the identification of criteria to demonstrate complete bidirectional block in patients undergoing cavotricuspid isthmus ablation for atrial flutter, the recurrence rate was about $30 \%$. After the description and the systematic use of these criteria, the recurrence rate has been reduced to $3 \%$.

However, even with complete PV isolation in patients with paroxysmal AF the procedure is associated with a $62-85 \%$ success rate without the use of antiarrhythmic drugs. ${ }^{9}$ The obvious question that remains is how to improve the current success rates of this procedure. Further improvements to the outcome will probably need further ablation to modify the atrial substrate. At the present time, the best strategy for substrate modification is unknown.

A variety of limited linear ablation strategies are being combined with PV ablation with improved outcomes. The systematic use of linear lesions in combination with PV ablation has recently been advocated by some groups. ${ }^{17} 18$ However, while the results are probably better, there has been no evidence to suggest that every patient with paroxysmal AF would benefit from linear ablation. In addition, linear ablation within the atria is associated with two important risks: (1) an increased procedural risk; and (2) a higher incidence of incisional macroreentry propagating through incomplete linear lesions. The latter complication has been seen in up to $20 \%$ of patients in the experience presented by Morady and colleagues at the Boston AF meeting (January 2004) using the anatomical approach without electrophysiological confirmation of conduction block. This strongly argues against a strategy of empirical linear lesions in all patients undergoing $\mathrm{AF}$ ablation, underscoring the importance of identifying the subset of patients who would benefit from such a strategy. At our centre, the deployment of linear lesions is restricted to patients with AF resistant to PV isolation, with either persistent or inducible sustained AF after PV isolation. Using this strategy, only $50 \%$ of the patients with paroxysmal AF require linear lesions and the overall success rate (without antiarrhythmics) at six months is $91 \%$.

\section{FUTURE OF CATHETER ABLATION FOR AF}

Recently, studies aiming to compare the efficacy of "rhythm versus rate control" in patients with AF have suggested that rate control is not inferior to rhythm control. ${ }^{19}{ }^{20}$ However, the results of these studies need to be interpreted in the context of their design; importantly, they were not designed to compare sinus rhythm versus AF but rather two different pharmacological strategies to manage AF. These studies have yet again demonstrated the limited efficacy of antiarrhythmic drugs in maintaining sinus rhythm and showed that pharmacological antiarrhythmic treatment was not superior to rate control. Importantly, these studies do not demonstrate that $\mathrm{AF}$ is as safe or as desirable as sinus rhythm.

Interestingly, in a recent presentation of an AFFIRM (atrial fibrillation follow-up investigation of rhythm management) substudy, Epstein and colleagues compared patients who could be maintained in sinus rhythm with those who had persistent $\mathrm{AF}$ and demonstrated a mortality risk reduction with the maintenance of sinus rhythm by $47 \%$, while the use of antiarrythmic drugs was associated with a $49 \%$ increased risk of death. ${ }^{21}$ Although not one of the predefined end points of the study, these results suggest that perhaps sinus rhythm is more desirable than AF and that it may be preferable to achieve this without the use of antiarrhythmic drugs. A recent single centre, non-randomised, observational report of patients undergoing AF ablation has provided further corroborative evidence in this respect, demonstrating improvement and return to baseline community mortality rates following AF ablation. ${ }^{22}$ In addition to the mortality benefit associated with AF ablation, accumulating evidence suggests expanding indications for non-pharmacologic strategies to maintain sinus rhythm. While these reports have been non-randomised and observational in design they have demonstrated prevention of prolonged sinus pauses and improvement in the quality of life after ablation to maintain sinus rhythm.

\section{CONCLUSION}

The last decade has seen significant developments in our understanding of $\mathrm{AF}$ and has led to catheter ablation techniques that have demonstrated the feasibility of achieving cure. As the maintenance of sinus rhythm without the use of antiarrhythmic drugs becomes more achievable in many patients (by many centres) it is likely that the indications for curative ablation of AF will expand. However, the efficacy of such a strategy of maintaining sinus rhythm to reduce the various complications associated with AF needs to be evaluated by clinical trials.

\section{Authors' affiliations \\ P Jais, P Sanders, L F Hsu, M Hocini, M Haissaguerre, Hopital \\ Cardiologique du Haut Leveque, Pessac, France}

\section{REFERENCES}

1 Haissaguerre M, Gencel L, Fischer B, et al. Successful catheter ablation of atrial fibrillation. J Cardiovasc Electrophysiol 1994;5:1045-52.

2 Swartz JF PG, Silvers J, Patten L, et al. A catheter-based curative approach to atrial fibrillation in humans [abstract]. Circulation 1994;90:I335.

3 Haissaguerre $M$, Jais $P$, Shah DC, et al. Right and left atrial radiofrequency catheter therapy of paroxysmal atrial fibrillation. J Cardiovasc Electrophysiol 1996; 7:1132-44

4 Jais $\mathbf{P}$, Haissaguerre $M$, Shah DC, et al. A focal source of atrial fibrillation treated by discrete radiofrequency ablation. Circulation 1997;95:572-6.

5 Haissaguerre $M$, Jais $P$, Shah DC, et al. Spontaneous initiation of atrial fibrillation by ectopic beats originating in the pulmonary veins. N Engl J Med 1998;339:659-66.

6 Marrouche NF, Dresing T, Cole C, et al. Circular mapping and ablation of the pulmonary vein for treatment of atrial fibrillation: impact of different catheter technologies. J Am Coll Cardiol 2002;40:464-74.

7 Pappone C, Oreto G, Rosanio S, et al. Atrial electroanatomic remodeling after circumferential radiofrequency pulmonary vein ablation: efficacy of an anatomic approach in a large cohort of patients with atrial fibrillation. Circulation 2001; 104:2539-44.

8 Chen SA, Tai CT, Hsieh MH, et al. Radiofrequency catheter ablation of atrial fibrillation initiated by spontaneous ectopic beats. Europace 2000;2:99-105.

9 Oral H, Knight BP, Ozaydin M, et al. Segmental ostial ablation to isolate the pulmonary veins during atrial fibrillation: feasibility and mechanistic insights. Circulation 2002;106:1256-62.

10 Oral H, Knight BP, Tada H, et al. Pulmonary vein isolation for paroxysmal and persistent atrial fibrillation. Circulation 2002;105:1077-81.

11 Bourke JP, Dunuwille A, O'Donnell D, et al. Pulmonary vein ablation for idiopathic atrial fibrillation: six month outcome of first procedure in 100 consecutive patients. Heart 2005;91:51-7. 
12 Kumagai $\mathrm{K}$, Yasuda $\mathrm{T}$, Tojo $\mathrm{H}$, et al. Role of rapid focal activation in the maintenance of atrial fibrillation originating from the pulmonary veins. Pacing Clin Electrophysiol 2000;11:1823-7.

13 O'Donnell D, Furniss SS, Bourke JP. Paroxysmal cycle length shortening in the pulmonary veins during atrial fibrillation correlates with arrhythmogenic triggering foci in sinus rhythm. J Cardiovasc Electrophysiol 2002;13:124-8.

14 Oral H, Ozaydin M, Tada H, et al. Mechanistic significance of intermittent pulmonary vein tachycardia in patients with atrial fibrillation. J Cardiovasc Electrophysiol 2002;13:645-50.

15 Haissaguerre $M$, Shah DC, Jais $P$, et al. Mapping-guided ablation of pulmonary veins to cure atrial fibrillation. Am J Cardiol 2000;86:K9-19.

16 Macle L, Jais P, Scavee C, et al. Pulmonary vein disconnection using the Localisa three-dimensional nonfluoroscopic catheter imaging system. J Cardiovasc Electrophysiol 2003;14:693-7.

17 Oral H, Scharf C, Chugh A, et al. Catheter ablation for paroxysmal atrial fibrillation: segmental pulmonary vein ostial ablation versus left atrial ablation. Circulation 2003; 108:2355-60.
18 Pappone C, Santinelli V, Manguso F, et al. Pulmonary vein denervation enhances long-term benefit after circumferential ablation for paroxysmal atrial fibrillation. Circulation 2004; 109:327-34.

19 Wyse DG, Waldo AL, DiMarco JP, et al. A comparison of rate control and rhythm control in patients with atrial fibrillation. N Engl J Med 2002;347:1825-33

20 Hagens VE, Van Gelder IC, Crijns HJ. The RACE study in perspective of randomized studies on management of persistent atrial fibrillation. Card Electrophysiol Rev 2003:7:118-21.

21 Epstein AE, Corley SD, the NHLBI AFFIRM Investigators. Relationship between sinus rhythm, treatment and survival in the atrial fibrillation follow-up investigation of rhythm management (AFFIRM) study [abstract]. Circulation 2003; 108:IV-472.

22 Pappone C, Rosanio S, Augello G, et al. Mortality, morbidity, and quality of life after circumferential pulmonary vein ablation for atrial fibrillation: outcomes from a controlled nonrandomized long-term study. J Am Coll Cardiol 2003;42: 185-97.

\section{IMAGES IN CARDIOLOGY}

\section{Renal tumour with cavoatrioventricular extension}

A

52 year old man was investigated for fever of unknown origin and a grade II/IV systolic murmur. The ECG and chest radiograph were normal. The patient had normocytic anaemia, moderate thrombocytosis, and elevated $\mathrm{C}$ reactive protein (CRP), alanine aminotransferase (ALT), and aspartate aminotransferase (AST). Investigations for collagen vascular disease were negative. An abdominal ultrasound revealed a $3 \times 3 \mathrm{~cm}$ mass in the anterosuperior aspect of the right kidney. Contrast enhanced abdominal tomography (panel A), and magnetic resonance imaging (panel B), showed an enlarged right kidney with inhomogeneous density, along with a $3 \mathrm{~cm}$ low density mass (arrow). Transthoracic (panel C) and transoesophageal (panels D and E) echocardiography disclosed a mobile echo-dense mass (arrow) protruding from the inferior vena cava (IVC) into the right atrium (RA) and through the tricuspid valve (TV) up to the right ventricular (RV) apex, creating tricuspid regurgitation without stenosis. Urgent surgical treatment was decided upon. The operation was done under deep hypothermia with circulatory arrest for 31 minutes. The intracardiac extension of the tumour was approached via longitudinal right atriotomy (panel F) and the whole mass was resected with the kidney, adrenal gland, and ipsilateral lymph nodes (panel $\mathrm{G})$. A patch of autologous pericardium was used for IVC closure. Extracorporeal circulation and patient rewarming started immediately after closure of the atrium. The patient had an uneventful course. Histological examination of the tumour disclosed clear cell carcinoma of the kidney (panel H), with infiltration of the renal vein, whereas the lymph nodes were not involved. One year later the patient remains disease-free.

\section{P A Davlouros A S Manolis E E Apostolakis asm@otenet.gr}
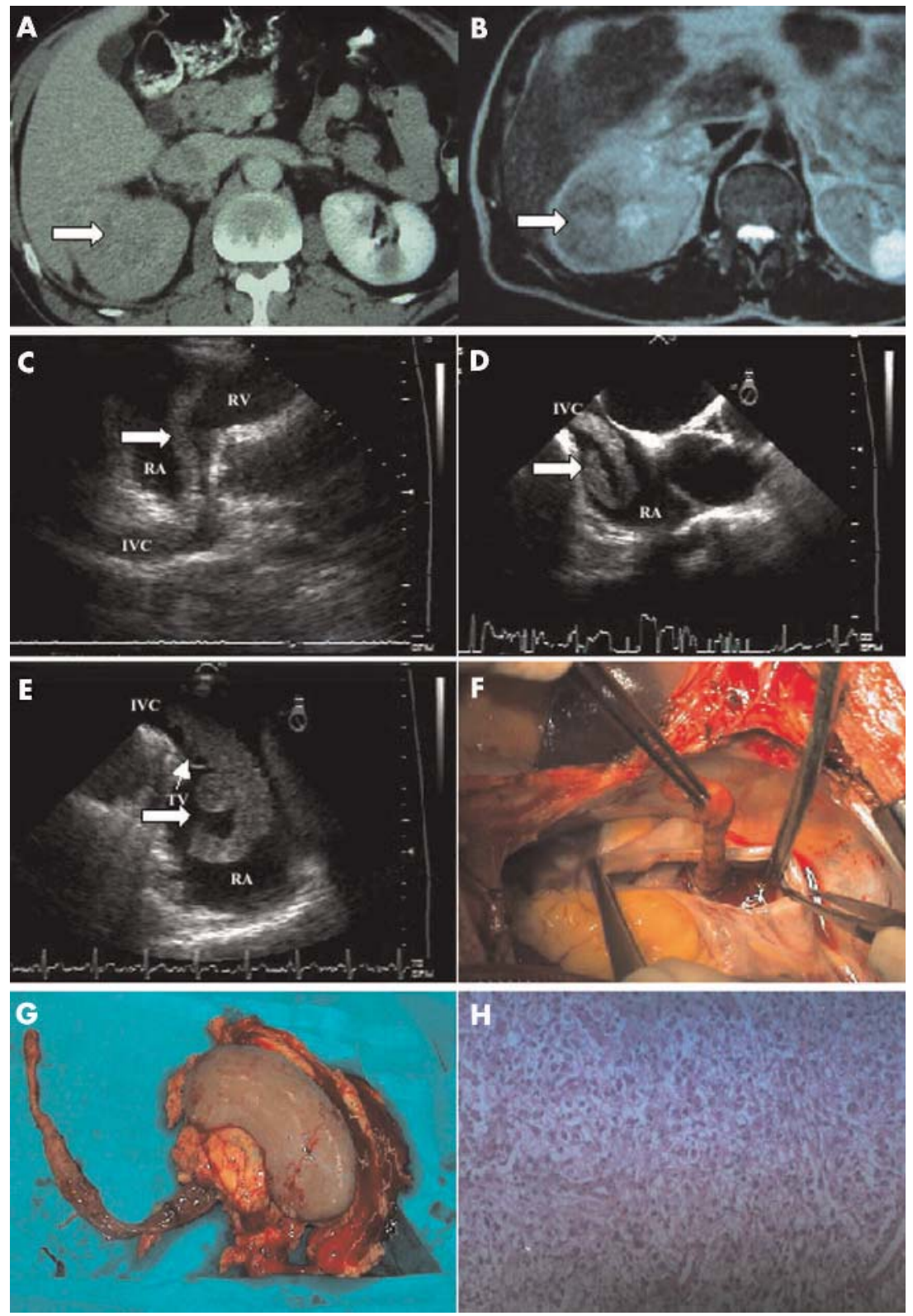